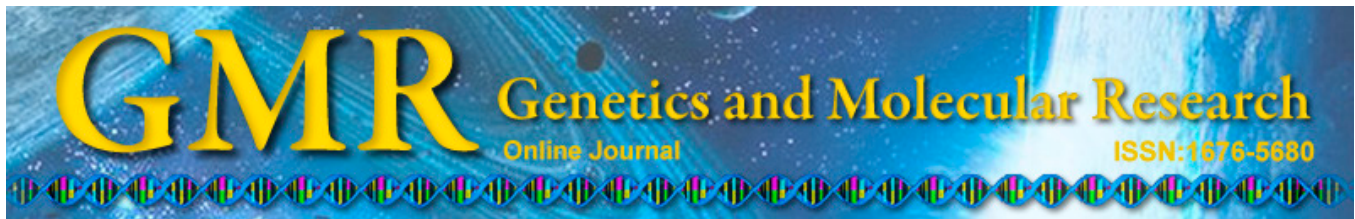

\title{
Meta-analysis of differentially expressed genes in autism based on gene expression data
}

\author{
L.F. Ning ${ }^{1,2}$, Y.Q. Yu ${ }^{1}$, E.T. GuoJi ${ }^{2}$, C.G. Kou ${ }^{1}$, Y.H. Wu ${ }^{1}$, J.P. Shi ${ }^{1}$, \\ L.Z. $\mathrm{Ai}^{1}$ and $\mathrm{Q}$. Yu ${ }^{1}$ \\ ${ }^{1}$ Department of Epidemiology and Biostatistics, School of Public Health, \\ Jilin University, Changchun, China \\ ${ }^{2}$ National Research Institute for Family Planning, Beijing, China \\ Corresponding author: Q. Yu \\ E-mail: lifeng_ningpre@163.com
}

Genet. Mol. Res. 14 (1): 2146-2155 (2015)

Received January 27, 2014

Accepted October 17, 2014

Published March 27, 2015

DOI http://dx.doi.org/10.4238/2015.March.27.1

\begin{abstract}
The purpose of this study was to identify differentially expressed (DE) genes and biological processes associated with changes in gene expression in autism. We performed a meta-analysis using new publicly available Gene Expression Omnibus (GEO) datasets of autism. We performed Gene Ontology (GO) enrichment analyses and pathway analysis using the Kyoto Encyclopedia of Genes and Genomes (KEGG). Ten GEO datasets, including 364 cases and 248 controls, were available for the meta-analysis. We identified 3105 genes that were consistently $\mathrm{DE}$ in autism (1425 upregulated and 1680 downregulated genes). We also found that 7 genes were associated with phospholipase A2 (PLA2), including LYPLA2P1, PLA2G4D, PNPLA2, LYPLA2, PLA2G6, PLA2G7, and PLA2G5. We found GO terms for molecular functions significantly enriched in structural constituent of ribosome (GO: 0003735, $\mathrm{P}=1.87$ E06) and transcription regulator activity (GO: 0030528, $\mathrm{P}=8.86 \mathrm{E}-04)$, while for biological processes, the enriched GO terms were involved in translational elongation (GO: 0006414, $\mathrm{P}=1.74 \mathrm{E}-12$ ) and the response to cytokine stimuli (GO: 0034097, $\mathrm{P}=2.76 \mathrm{E}-05)$. The most significant pathway in our KEGG analysis was the ribosome pathway $(\mathrm{P}=7.90 \mathrm{E}-$
\end{abstract}


12). Our meta-analysis identified genes that were consistently DE and biological pathways associated with gene expression changes in autism.

Key words: Autism; Differentially expressed genes; Expression data; Meta-analysis; Microarray

\section{INTRODUCTION}

Autism is a common, highly heritable neuro-developmental condition characterized by marked genetic heterogeneity (Durand et al., 2007). Various etiologies have been suggested for this complex syndrome. Though it is associated with a high degree of heritability, the specific genes responsible for autism remain unknown. Various other factors have been implicated, including immunological (Ashwood et al., 2006), neurological (Hashimoto et al., 1993), and environmental (London, 2000) factors. Thus, a fundamental question is whether autism represents an etiologically heterogeneous disorder in which a myriad genetic or environmental risk factors perturb common underlying molecular pathways in the brain (Geschwind, 2008). However, in contrast with many other brain disorders, including neurodegenerative diseases such as Parkinson's or Alzheimer's diseases, autism lacks a clear unifying pathology at the molecular, cellular, and systems levels.

Emerging evidence implies that abnormal fatty acid metabolism plays a contributing role in autism pathology. Recent literature suggests that fatty acid homeostasis may be altered in autism as a result of insufficient dietary supplementation, genetic defects, function of enzymes involved in their metabolism, or influence of various environmental agents such as infections, inflammation, or drugs. Phospholipase A2 (PLA2) is an enzyme involved in maintaining membrane phospholipids. There are 3 major types of PLA2 enzymes: the calcium-dependent group IV cytosolic PLA2, the group II secretory PLA2, and the group VI calcium-independent PLA2 (Sun et al., 2004). Elevated levels of PLA2 in red blood cells have been associated with neuropsychiatric disorders such as schizophrenia, depression, bipolar disorder, dyslexia, and autism (Horrobin and Bennett, 1999; Bell et al., 2004).

Determining global levels of gene expression regulation may be particularly important for understanding the pathological basis of diseases such as autism, in which multiple systems are affected. Alterations at the global level of gene expression regulation may be shared across systems, even when the tissue-specific genes affected by these global changes differ. Highthroughput genomics technologies have improved our understanding of the complex interactions and networks involved in disease development. Microarrays measure the expression of thousands of genes simultaneously on a genome-wide scale (Golub et al., 1999). Alterations in gene profiles can be correlated to altered gene functions and biochemical activities. Microarray is a powerful tool that has rapidly increased as an investigational method in medical research.

Identification of gene expression signatures that differentiate the disease state from healthy controls is dependent on samples, sample size, heterogeneous data sets, and reproducibility (Ramasamy et al., 2008). Although many microarray studies have provided lists of differentially expressed (DE) genes, there are inconsistencies among studies resulting from limitations such as small sample sizes (Siddiqui et al., 2006). Data integration techniques, such as meta-analyses, combine available data and integrate information from multiple independent but related microarray studies to identify significant genes (Feichtinger et al., 2012). Combining data from various studies can enhance the reliability and generalizability of the results (Ramasamy et al., 2008) and can be used to more precisely estimate gene expression. In 
particular, the benefit of increased statistical power can help to overcome the limitation of microarray studies. In this study, we used a new method to perform a meta-analysis of expression data of multiple gene expression data sets. To overcome the limitations of individual studies, resolve inconsistencies, and reduce the likelihood of random errors revealing false-positive or false-negative associations, we performed a microarray meta-analysis to identify DE genes and biological processes associated with gene expression changes in autism.

\section{MATERIAL AND METHODS}

\section{Identification of eligible autism}

\section{ASD gene expression datasets}

We first queried PubMed and related databases for expression profiling studies comparing autism and normal control tissues (NT). The following key words and their combinations were used: "autism, gene expression, microarray, genetics". In addition, the Gene Expression Omnibus database (GEO, http://www.ncbi.nlm.nih.gov/geo) was also searched to identify any other relevant studies. The search results were limited to those published between 2000 and March 2013. We only retained the original experimental articles that analyzed gene expression profiling of autism and NT. Non-human studies, review articles, and integrated analysis of expression profiles were excluded. We conducted this meta-analysis in accordance with the guidelines provided in the PRISMA statement (The PRISMA Checklist S1). Data were extracted from the original studies by 2 independent reviewers. Any discrepancies between reviewers were resolved by consensus or consultation with a third reviewer. The following information was extracted from each identified study: GEO accession number, sample type, platform, number of cases and controls, references, and gene expression data.

\section{Data preprocessing}

Normalization is important for comparing microarray data sets. The heterogeneity of different datasets caused by using different platforms, different gene nomenclature, and different control tissues may make it difficult to directly compare studies. Thus, a global normalization method for minimizing inconsistency should be used. For this purpose, we used the Z-score transformation approach to calculate the expression intensities for each probe of the gene expression profiles. Z-scores were calculated according to the formula:

$$
\text { Z score }=\frac{x_{i}-\bar{x}}{\delta}
$$

where $\mathrm{x}_{\mathrm{i}}$ represents raw intensity data for each gene; $\overline{\mathrm{x}}$ represents average gene intensity within a single experiment, and $\delta$ represents standard deviation (SD) of all measured intensities.

\section{Statistical analysis}

The significance analysis of the microarray software was then used to identify the DE genes between disease and control samples. This procedure combines the calculation of a Student $t$-test statistic value for each gene with subsequent permutation analysis and false 
discovery rate (FDR) calculation. To obtain the best balance between the number of significant calls and the lowest FDR for the dataset tested, we selected genes showing at least 2-fold changes and an FDR less than 0.05 as significantly DE.

\section{Functional classification of DE genes}

To examine the biological significance of DE genes, we performed Gene Ontology (GO) enrichment analysis to investigate their functional distribution. The online-based software GeneCoDis3 (http://genecodis.cnb.csic.es) was used for this analysis. Fisher's exact test was used at $\mathrm{P}$ value $<0.1$ and significance level was adjusted by a correction for FDR. In addition, we also performed pathway enrichment analysis based on the Kyoto Encyclopedia of Genes and Genomes (KEGG) database.

\section{RESULTS}

\section{Overview of the studies included}

In this study, we identified a total of 10 expression-profiling studies based on our inclusion criteria, which included 612 samples of 364 cases and 248 controls. The characteristics of studies included in this analysis are listed in Table 1 (Nishimura et al., 2007; Gregg et al., 2008; Hu et al., 2009a,b; Alter et al., 2011; Voineagu et al., 2011; Kuwano et al., 2011; Ginsberg et al., 2012). The data sets for GSE30573 and GSE28521 were examined in the same study, but GSE30573 was RNA - seqdata set, and the data sets we applied were almost microarray data sets except for GSE30573.

Table 1. Characteristics of individual studies included in the meta-analysis.

\begin{tabular}{llcl}
\hline GEO ID & Sample source & Numbers (AU:NT) & Platform \\
\hline GSE7329 & Blood & $15: 15$ & GPL1708 Agilent-012391 G4112A \\
GSE6575 & Blood & $49: 12$ & GPL570 AffymetrixU133 plus 2.0 \\
GSE15451 & Blood & $21: 17$ & GPL3427 TIGR 40K \\
GSE15402 & Blood & $87: 29$ & GPL3427 TIGR 40K \\
GSE26415 & Blood & $42: 42$ & GPL6480 Agilent-014850 4x44K G4112F \\
GSE28521 & Brain & $40: 39$ & GPL6883 Illumina HumanRef-8 v3.0 expression beadchip \\
GSE30573 & Brain & $3: 3$ & GPL9115 Illumina Genome Analyzer II \\
GSE25507 & Blood & $82: 64$ & GPL570 AffymetrixU133 plus 2.0 \\
GSE38609 & Brain & $9: 9$ & GPL10558 Illumina HumanHT-12 V4.0 expression beadchip \\
GSE43076 & Blood & $16: 18$ & GPL6480 Agilent-014850 4x44K G4112F \\
\hline
\end{tabular}

$\mathrm{AU}=$ Autism; $\mathrm{NT}=$ normal tissue.

\section{Global changes in gene expression}

To identify genetic markers involved in the development and progression of child autism, we determined the probe ID, for a microarray platform that represent a named gene, and the HUGO symbol of that gene, for each data set to the National Center for Biotechnology Information (NCBI) gene ID. The expression value was logarithmically transformed (base 2) and then transformed to a Z-score for global normalization. After filtering the normalized data, 33,502 genes were detected in more than $60 \%$ of the samples. 
Using the assembled expression compendium, we investigated global shifts in gene expression between child autism and corresponding NTs, respectively. The significance analysis of the microarray method was used to identify DE genes between pathological and control samples. With the threshold of FDR $<0.05$ and minimal 2-fold changes, a total of 3105 genes were found to be DE between child autism and NT. Among the 3105 DE genes, 1425 genes were upregulated and 1680 genes were downregulated. Lists of the top 20 most significantly up- or downregulated genes are provided in Tables 2 and 3, respectively. We also identified 7 genes associated with PLA2, including LYPLA2P1, PLA2G4D, PNPLA2, LYPLA2, PLA2G6, PLA2G7, and PLA2G5 (Table 4).

Table 2. Top 20 upregulated differentially expressed genes.

\begin{tabular}{rlccl}
\hline Gene ID & Gene symbol & Combined ES & P value & Gene name \\
\hline 124975 & GGT6 & $1.06 \mathrm{E}-02$ & $2.60 \mathrm{E}-09$ & Gamma-glutamyltransferase 6 \\
7475 & WNT6 & $1.35 \mathrm{E}-02$ & $2.62 \mathrm{E}-09$ & Wingless-type MMTV integration site family, member 6 \\
84214 & DKFZP434F142 & $1.70 \mathrm{E}+01$ & $2.92 \mathrm{E}-09$ & Uncharacterized DKFZp434F142 \\
283651 & HMGN2P46 & $3.69 \mathrm{E}-02$ & $3.01 \mathrm{E}-09$ & High mobility group nucleosomal binding domain 2 pseudogene 46 \\
678 & ZFP36L2 & $1.91 \mathrm{E}-02$ & $4.95 \mathrm{E}-09$ & ZFP36 ring finger protein-like 2 \\
8352 & HIST1H3C & $2.10 \mathrm{E}+01$ & $5.97 \mathrm{E}-09$ & Histone cluster 1, H3c \\
3126 & HLA-DRB4 & $1.45 \mathrm{E}+01$ & $7.01 \mathrm{E}-09$ & Major histocompatibility complex, class II, DR beta 4 \\
8350 & HIST1H3A & $1.78 \mathrm{E}+00$ & $1.22 \mathrm{E}-08$ & Histone cluster 1, H3a \\
129880 & BBS5 & $9.34 \mathrm{E}+00$ & $1.38 \mathrm{E}-08$ & Bardet-Biedl syndrome 5 \\
2837 & UTS2R & $2.03 \mathrm{E}-02$ & $1.59 \mathrm{E}-08$ & Urotensin 2 receptor \\
3045 & HBD & $9.67 \mathrm{E}-02$ & $1.63 \mathrm{E}-08$ & Hemoglobin, delta \\
963 & CD53 & $2.00 \mathrm{E}+01$ & $1.65 \mathrm{E}-08$ & CD53 molecule \\
8290 & HIST3H3 & $4.19 \mathrm{E}-03$ & $1.67 \mathrm{E}-08$ & Histone cluster 3, H3 \\
339665 & SLC35E4 & $2.83 \mathrm{E}-02$ & $1.78 \mathrm{E}-08$ & Solute carrier family 35, member E4 \\
84757 & MGC10814 & $6.11 \mathrm{E}-02$ & $1.88 \mathrm{E}-08$ & Uncharacterized protein MGC10814 \\
6181 & RPLP2 & $2.06 \mathrm{E}-01$ & $1.97 \mathrm{E}-08$ & Ribosomal protein, large, P2 \\
79955 & PDZD7 & $1.95 \mathrm{E}-02$ & $2.03 \mathrm{E}-08$ & PDZ domain containing 7 \\
147700 & KLC3 & $3.49 \mathrm{E}-02$ & $2.05 \mathrm{E}-08$ & Kinesin light chain 3 \\
8717 & TRADD & $5.57 \mathrm{E}+04$ & $2.14 \mathrm{E}-08$ & TNFRSF1A-associated via death domain \\
613126 & LOC613126 & $7.33 \mathrm{E}-02$ & $2.19 \mathrm{E}-08$ & Uncharacterized LOC613126 \\
\hline
\end{tabular}

$\mathrm{ES}=$ effect size.

Table 3. Top 20 downregulated differentially expressed genes.

\begin{tabular}{rllll}
\hline Gene ID & Gene symbol & Combined ES & P value & Gene name \\
\hline 283849 & EXOC3L1 & 0.839180474 & 0.0088416 & Exocyst complex component 3-like 1 \\
677821 & SNORA39 & 0.576012831 & 0.0041636 & Small nucleolar RNA, H/ACA box 39 \\
55716 & LMBR1L & 0.81735657 & 0.0079 & Limb development membrane protein 1-like \\
693187 & MIR602 & 0.825906322 & 0.001643 & MicroRNA 602 \\
90525 & SHF & 0.562686734 & 0.0092982 & Src homology 2 domain containing F \\
339122 & RAB43 & 0.82111752 & 0.0083847 & RAB43, member RAS oncogene family \\
10984 & KCNQ1OT1 & 0.790779915 & 0.00171585 & KCNQ1 opposite strand/antisense transcript 1 (non-protein coding) \\
390260 & OR6X1 & 0.851178751 & 0.0064821 & Olfactory receptor, family 6, subfamily X, member 1 \\
650683 & LOC650683 & 0.740192555 & 0.0067242 & Hypothetical protein LOC650683 \\
386676 & KRTAP10-9 & 0.809996476 & 0.0079572 & Keratin associated protein 10-9 \\
641804 & LOC641804 & 0.78233216 & 0.0041796 & Similar to GTF2I repeat domain containing 1 isoform 2 \\
256892 & OR51F1 & 0.737303349 & 0.006725 & Olfactory receptor, family 51, subfamily F, member 1 \\
344657 & LRRIQ4 & 0.845407404 & 0.0093379 & Leucine-rich repeats and IQ motif containing 4 \\
3737 & KCNA2 & 0.650380382 & 0.0097038 & Potassium voltage-gated channel, shaker-related subfamily, member 2 \\
284613 & CYB561D1 & 0.795587453 & 0.0071833 & Cytochrome b561 family, member D1 \\
9820 & CUL7 & 0.768716592 & 0.0063811 & Cullin 7 \\
8659 & ALDH4A1 & 0.801344254 & 0.0027847 & Aldehyde dehydrogenase 4 family, member A1 \\
79228 & THOC6 & 0.373313212 & 0.0081636 & THO complex 6 homolog (Drosophila) \\
64946 & CENPH & 0.804828762 & 0.0079593 & Centromere protein H \\
5877 & RABIF & 0.793222926 & 0.0056414 & RAB interacting factor \\
\hline
\end{tabular}


Table 4. PLA2-associated differentially expressed genes.

\begin{tabular}{rlll}
\hline Gene ID & Gene symbol & P value & Gene name \\
\hline 285840 & LYPLA2P1 & $8.24 \mathrm{E}-03$ & Lysophospholipase II pseudogene 1 \\
283748 & PLA2G4D & $1.36 \mathrm{E}-03$ & Phospholipase A2, group IVD (cytosolic) \\
57104 & PNPLA2 & $5.58 \mathrm{E}-03$ & Patatin-like phospholipase domain containing 2 \\
11313 & LYPLA2 & $1.02 \mathrm{E}-03$ & Lysophospholipase II \\
8398 & PLA2G6 & $8.52 \mathrm{E}-03$ & Phospholipase A2, group VI (cytosolic, calcium-independent) \\
7941 & PLA2G7 & $3.62 \mathrm{E}-03$ & Phospholipase A2, group VII (platelet-activating factor acetylhydrolase, plasma) \\
5322 & PLA2G5 & $8.95 \mathrm{E}-04$ & Phospholipase A2, group V \\
\hline
\end{tabular}

\section{Functional annotation of DE genes}

To gain insight into the biological roles of these DE genes, we performed GO categories enrichment analysis. GO provides a common descriptive framework and functional annotation and classification for gene sets analysis. GO categories are organized into 3 groups: biological process, molecular function, and cellular component. In our study, only the biological process and molecular function categories were considered. Using an FDR $<0.01$ threshold, we identified 146 significant enrichments for DE genes. We identified GO terms for molecular functions significantly enriched in structural constituent of ribosome (GO: 0003735, P = 1.87E06) and transcription regulator activity (GO: 0030528, $\mathrm{P}=8.86 \mathrm{E}-04$ ) (Table 5), while for biological processes, the enriched GO terms were translational elongation (GO: 0006414, $\mathrm{P}=$ 1.74E-12) and response to cytokine stimulus (GO: 0034097, $\mathrm{P}=2.76 \mathrm{E}-05$ ).

\begin{tabular}{|c|c|c|}
\hline GO ID & Term & $P$ value \\
\hline \multicolumn{3}{|c|}{ Molecular functions } \\
\hline GO: 0003735 & Structural constituent of ribosome & $1.87 \mathrm{E}-06$ \\
\hline GO: 0030528 & Transcription regulator activity & $8.86 \mathrm{E}-04$ \\
\hline GO: 0008134 & Transcription factor binding & $9.53 \mathrm{E}-04$ \\
\hline GO: 0032393 & MHC class I receptor activity & $3.33 \mathrm{E}-03$ \\
\hline GO: 0003723 & RNA binding & 4.01E-03 \\
\hline GO: 0043565 & Sequence-specific DNA binding & $4.03 \mathrm{E}-03$ \\
\hline GO: 0016563 & Transcription activator activity & $4.31 \mathrm{E}-03$ \\
\hline GO: 0003700 & Transcription factor activity & $4.39 \mathrm{E}-03$ \\
\hline GO: 0030274 & LIM domain binding & $6.76 \mathrm{E}-03$ \\
\hline GO: 0003950 & NAD + ADP-ribosyltransferase activity & $1.02 \mathrm{E}-02$ \\
\hline GO: 0003677 & DNA binding & $1.21 \mathrm{E}-02$ \\
\hline GO: 0003712 & Transcription cofactor activity & $1.44 \mathrm{E}-02$ \\
\hline GO: 0005254 & Chloride channel activity & $1.79 \mathrm{E}-02$ \\
\hline GO: 0005344 & Oxygen transporter activity & $1.80 \mathrm{E}-02$ \\
\hline GO: 0016776 & Phosphotransferase activity, phosphate group as acceptor & $1.96 \mathrm{E}-02$ \\
\hline \multicolumn{3}{|c|}{ Biological processes } \\
\hline GO: 0006414 & Translational elongation & $1.74 \mathrm{E}-12$ \\
\hline GO: 0034097 & Response to cytokine stimulus & $2.76 \mathrm{E}-05$ \\
\hline GO: 0006412 & Translation & $3.39 \mathrm{E}-05$ \\
\hline GO: 0045058 & $\mathrm{~T}$ cell selection & $2.36 \mathrm{E}-04$ \\
\hline GO: 0015908 & Fatty acid transport & $3.65 \mathrm{E}-04$ \\
\hline GO: 0006325 & Chromatin organization & $1.10 \mathrm{E}-03$ \\
\hline GO: 0051276 & Chromosome organization & $1.73 \mathrm{E}-03$ \\
\hline GO: 0045449 & Regulation of transcription & $1.82 \mathrm{E}-03$ \\
\hline GO: 0046649 & Lymphocyte activation & $2.01 \mathrm{E}-03$ \\
\hline GO: 0042110 & $\mathrm{~T}$ cell activation & $2.02 \mathrm{E}-03$ \\
\hline GO: 0006916 & Anti-apoptosis & $2.16 \mathrm{E}-03$ \\
\hline GO: 0006333 & Chromatin assembly or disassembly & $2.28 \mathrm{E}-03$ \\
\hline GO: 0043067 & Regulation of programmed cell death & $2.68 \mathrm{E}-03$ \\
\hline GO: 0043069 & Negative regulation of programmed cell death & $2.93 \mathrm{E}-03$ \\
\hline GO: 0015909 & Long-chain fatty acid transport & $2.94 \mathrm{E}-03$ \\
\hline
\end{tabular}


To further evaluate the biological significance of the DE genes, we also performed a KEGG pathway enrichment analysis (Table 6). The most significant pathway in our KEGG analysis was the ribosome pathway $(\mathrm{P}=7.90 \mathrm{E}-12)$. Furthermore, graft-versus-host disease $(\mathrm{P}=9.67 \mathrm{E}-$ 03 ) and primary immunodeficiency pathways $(\mathrm{P}=3.32 \mathrm{E}-02)$ were found to be highly enriched.

\begin{tabular}{|c|c|c|}
\hline KEGG pathway & $P$ value & Genes \\
\hline Ribosome & $7.90 \mathrm{E}-12$ & $\begin{array}{l}\text { LOC729362, LOC441073, LOC728139, LOC644464, RPS11, LOC388339, RPL374, } \\
\text { LOC100129685, RPL38, LOC644934, RPL14, RPS4X, LOC647285, LOC728576, LOC645174, } \\
\text { LOC646766, LOC646966, LOC } 389156, \text { RPL27A, LOC643358, LOC100131205, LOC100129882, } \\
\text { RPLPOP2, SNORA7B, RPS19, LOC387867, RPL12P6, RPS4X, LOC729402, RPS6, LOC400652, } \\
\text { RPLP2, LOC100128060, RPL18, LOC648729, LOC100129902, LOC389141, LOC730029, } \\
\text { LOC389342, LOC440575, LOC642892, RPS10P7, LOC100129424, LOC388556, LOC653156, } \\
\text { RPL13, RPL10, LOC440737, RPL7A, LOC100128936, LOC728782, RPS27, LOC388474, } \\
\text { LOC391833, RPL10L, RPS21, LOC653737, LOC441034 }\end{array}$ \\
\hline Graft-versus-host disease & $9.67 \mathrm{E}-03$ & $\begin{array}{l}\text { HLA-DRB4, KLRD1, KIR3DL1, CD28, KIR2DL2, KIR2DL3, PRF1, GZMB, HLA-B, } \\
\text { IL1B, HLA-F, HLA-E, HLA-DRB1 }\end{array}$ \\
\hline Primary immunodeficiency & $3.32 \mathrm{E}-02$ & CD4OLG, CD4, CD40, CIITA, UNG RFXAP, CD3D, CD3E, CD8B, CD79A \\
\hline
\end{tabular}

\section{DISCUSSION}

Various genes are DE in individuals with autism, and identifying the most important genes and pathways associated with the disease is very important. We used a meta-analysis approach of DE genes from microarray datasets to identify genes that were consistently DE at a statistically significant level, and performed GO enrichment analysis and pathway analysis using KEGG.

We performed a meta-analysis using 10 publicly available GEO data sets to identify common biological mechanisms involved in the pathogenesis of autism. Nine of these GEO data sets were based on microarray data, while the GSE30573 dataset was from RNA sequencing determined in the same study as GSE28521. In 3 studies, postmortem brain tissue from individuals with autism was analyzed to show that the glutamate neurotransmitter system is altered in patients with autism, while other studies examined blood cells from autistic individuals. We identified genes that were consistently up- or downregulated, showed significant GO enrichment, and pathways associated with autism. A total of3105 genes across the studies were consistently DE in autism (1425 upregulated and 1680 downregulated). Among these 3105 DE genes, 7 genes were associated with PLA2. To identify the biological processes associated with gene expression changes in autism, we performed GO analysis for the DE genes. The most significant enrichment among the list of molecular functions was the GO category of structural constituents of the ribosome $(\mathrm{P}=1.87-\mathrm{E} 06)$. Other significant $\mathrm{GO}$ categories for biological processes included translational elongation $(\mathrm{P}=1.74 \mathrm{E}-12)$ and the response to cytokine stimulus $(\mathrm{P}=2.76 \mathrm{E}-05)$. The most significant pathway in our KEGG analysis was ribosome pathway $(\mathrm{P}=7.90 \mathrm{E}-12)$. Furthermore, the graft-versus-host disease $(\mathrm{P}=9.67 \mathrm{E}-03)$ and primary immunodeficiency pathways $(\mathrm{P}=3.32 \mathrm{E}-02)$ were found to be highly enriched.

Using genome-wide differential display approaches, a number of recent studies have highlighted single-nucleotide polymorphisms, copy number variants, and epigenetic factors involved in the dysregulated expression of candidate genes related to autism occurrence (Bill and Geschwind, 2009). Putative and known candidates contributing to autism susceptibility are categorized based on the differentiation of neurons (e.g., DISC1, MET, PTEN, and ITGB3), 
neuronal cell adhesion (e.g., $N R X N 1, N L G N 3$, and $N L G N 4 X$ ), transmission of nervous system (e.g., OXTR, SLC6A4, GABRB3, and SHANK3), and regulation of neuronal activity (e.g., $F M R 1, M E C P 2$, and UBE3A) (Ingram et al., 2000; Martin et al., 2000; Wassink et al., 2001; Jamain et al., 2002; Campbell et al., 2006; Hu et al., 2006; Talebizadeh et al., 2006; Durand et al., 2007). In this study we identified autism susceptibility genes such as WNT2, EGR2, HOXA1, and SHAK3 among the 3105 differentially expressed genes. One of the underlying biological components of neuro-developmental disorders may involve dysregulation of phospholipid metabolism. Therefore, we also identified genes associated with PLA2, including LYPLA2P1, PLA2G4D, PNPLA2, LYPLA2, PLA2G6, PLA2G7, and PLA2G5, among these DE genes identified. Evidence has accumulated regarding elevated plasma levels of PLA2 in schizophrenia patients compared with healthy controls. Three single-nucleotide polymorphisms in the gene encoding for cytosolic PLA2 have been linked to schizophrenia and were found to play a role in the etiology of this disorder (Wei et al., 1998; Tao et al., 2005). Interestingly, the genes encoding human calcium-independent PLA2 and secretory PLA2 map to regions on chromosome 8q2324 and 7q31, respectively (Meyer et al., 1996), which have been previously linked to autism (Chen et al., 2005; Combi et al., 2010). This suggests that the altered levels of arachidonic acid and docohexaenoic acid in individuals with autism may be attributed to abnormalities in PLA2. Indeed, significantly increased activity of type IV PLA2 has been reported in the red blood cells of patients with autism and Asperger's syndrome compared to controls, strengthening the hypothesis that abnormal lipid metabolism occurs in autism (Bell et al., 2004, 2010). The increased PLA2 activity in individuals with autism may cause elevated breakdown of polyunsaturated fatty acids and their subsequent reduced incorporation into membrane phospholipids. Overall, the literature suggests a link between abnormalities in PLA2 enzymes and some psychiatric disorders, including autism spectrum disorders, which substantiates the importance of downstream lipid signaling molecules in proper nervous system functioning.

Interestingly, the most significant pathway in our KEGG analysis was the ribosome pathway. Ribosomal proteins play a crucial role in the regulation of protein synthesis. Therefore, their expression must be strictly controlled (Caldarola et al., 2009). This finding suggests a generalized and non-targeted process and may be a result of deregulation of other pathways. Several lines of evidence have identified a link between ribosome biogenesis and diseases such as cancer, anemia, and aging. A recent review also emphasized that protein synthesis is tightly linked to the regulation of neurological processes and cell growth (Twiss and Fainzilber, 2009). Deregulation of genes encoding ribosomal proteins may indirectly reflect an atypical process of neurological development in subjects with autism.

There were some limitations to our study. First, heterogeneity and confounding factors may have distorted our analysis. Clinical samples may be heterogeneous with respect to clinical activity, severity, or gender. Second, there were differences in gene expression between tissues such as the blood and brain that were not taken into account. However, our meta-analysis integrated data from different studies, enabling us to detect genes that would otherwise not have been identified. Despite these limitations, our findings have important implications for the pathophysiology of autism.

\section{REFERENCES}

Alter MD, Kharkar R, Ramsey KE, Craig DW, et al. (2011). Autism and increased paternal age related changes in global levels of gene expression regulation. PLoS One 6: e16715. 
Ashwood P, Wills S and Van de Waer J (2006). The immune response in autism: a new frontier for autism research. $J$. Leukoc. Biol. 80: 1-15.

Bell JG, MacKinlay EE, Dick JR, MacDonald DJ, et al. (2004). Essential fatty acids and phospholipase A2 in autistic spectrum disorders. Prostaglandins Leukot. Essent. Fatty Acids 71: 201-204.

Bell JG, Miller D, MacDonald DJ, MacKinlay EE, et al. (2010). The fatty acid compositions of erythrocyte and plasma polar lipids in children with autism, developmental delay or typically developing controls and the effect of fish oil intake. Br. J. Nutr. 103: 1160-1167.

Bill BR and Geschwind DH (2009). Genetic advances in autism: heterogeneity and convergence on shared pathways. Curr. Opin. Genet. Dev. 19: 271-278.

Caldarola S, De Stefano MC, Amaldi F and Loreni F (2009). Synthesis and function of ribosomal proteins-fading models and new perspectives. FEBS J. 276: 3199-3210.

Campbell DB, Sutcliffe JS, Ebert PJ, Militerni R, et al. (2006). A genetic variant that disrupts MET transcription is associated with autism. Proc. Natl. Acad. Sci. U. S. A. 103: 16834-16839.

Chen GK, Kono N, Geschwind DH and Cantor RM (2005). Quantitative trait locus analysis of nonverbal communication in autism spectrum disorder. Mol. Psychiatry 11: 214-220.

Combi R, Redaelli S, Beghi M, Clerici M, et al. (2010). Clinical and genetic evaluation of a family showing both autism and epilepsy. Brain Res. Bull. 82: 25-28.

Durand CM, Betancur C, Boeckers TM, Bockman J, et al. (2007). Mutations in the gene encoding the synaptic scaffolding protein SHANK3 are associated with autism spectrum disorders. Nat. Genet. 39: 25-27.

Feichtinger J, Thallinger GG, McFarlane RJ and Larcombe LD (2012). Microarray Meta-Analysis: From Data to Expression to Biological Relationships. In: Computational Medicine. Springer, New York, 59-77.

Geschwind DH (2008). Autism: many genes, common pathways? Cell 135: 391-395.

Ginsberg MR, Rubin RA, Falcone T, Ting AH, et al. (2012). Brain transcriptional and epigenetic associations with autism. PloS One 7: e44736.

Golub TR, Slonim DK, Tamayo P, Huard C, et al. (1999). Molecular classification of cancer: class discovery and class prediction by gene expression monitoring. Science 286: 531-537.

Gregg JP, Lit L, Baron CA, Hertz-Picciottto I, et al. (2008). Gene expression changes in children with autism. Genomics 91: 22-29.

Hashimoto T, Tayama M, Miyazaki M, Murakawa K, et al. (1993). Brainstem and cerebellar vermis involvement in autistic children. J. Child. Neurol. 8: 149-153.

Horrobin DF and Bennett CN (1999). New gene targets related to schizophrenia and other psychiatric disorders: enzymes, binding proteins and transport proteins involved in phospholipid and fatty acid metabolism. Prostaglandins Leukot. Essent. Fatty Acids 60: 141-167.

Hu VW, Frank BC, Heine S, Lee NH, et al. (2006). Gene expression profiling of lymphoblastoid cell lines from monozygotic twins discordant in severity of autism reveals differential regulation of neurologically relevant genes. BMC Genomics 7: 118.

Hu VW, Nguyen A, Kim KS, Steinberg ME, et al. (2009a). Gene expression profiling of lymphoblasts from autistic and nonaffected sib pairs: altered pathways in neuronal development and steroid biosynthesis. PloS One 4: e5775.

$\mathrm{Hu}$ VW, Sarachana T, Kim KS, Nguyen A, et al. (2009b). Gene expression profiling differentiates autism case-controls and phenotypic variants of autism spectrum disorders: evidence for circadian rhythm dysfunction in severe autism. Autism Res. 2: 78-97.

Ingram JL, Stodgell CJ, Hyman SL, Figlewicz DA, et al. (2000). Discovery of allelic variants of HOXA1 and HOXB1: genetic susceptibility to autism spectrum disorders. Teratology 62: 393-405.

Jamain S, Betancur C, Quach H, Philippe A, et al. (2002). Linkage and association of the glutamate receptor 6 gene with autism. Mol. Psychiatry 7: 302-310.

Kuwano Y, Kamio Y, Kawai T, Katsuura S, et al. (2011). Autism-associated gene expression in peripheral leucocytes commonly observed between subjects with autism and healthy women having autistic children. PloS One 6: e24723.

London EA (2000). The environment as an etiologic factor in autism: a new direction for research. Environ. Health Perspect. 108: 401-404.

Martin ER, Menold MM, Wolpert CM, Bass MP, et al. (2000). Analysis of linkage disequilibrium in gamma-aminobutyric acid receptor subunit genes in autistic disorder. Am. J. Med. Genet. 96: 43-48.

Meyer AH, Schmuck K, Morel C, Wishart W, et al. (1996). Localization of a gene coding for the phospholipase A2-L subtype (PLA2L) to human chromosome 8q24-qter. Genomics 38: 435-437.

Nishimura Y, Martin CL, Vazquez-Lopez A, Spence SJ, et al. (2007). Genome-wide expression profiling of lymphoblastoid cell lines distinguishes different forms of autism and reveals shared pathways. Hum. Mol. Genet. 16: 1682-1698.

Ramasamy A, Mondry A, Holmes CC and Altman DG (2008). Key issues in conducting a meta-analysis of gene expression

Genetics and Molecular Research 14 (1): 2146-2155 (2015)

CFUNPEC-RP www.funpecrp.com.br 
microarray datasets. PLoS Med. 5: e184.

Siddiqui AS, Delaney AD, Schnerch A, Griffith OL, et al. (2006). Sequence biases in large scale gene expression profiling data. Nucleic Acids Res. 34: e83-e83.

Sun GY, Xu J, Jensen MD and Simonyi A (2004). Phospholipase A2 in the central nervous system: implications for neurodegenerative diseases. J. Lipid Res. 45: 205-213.

Talebizadeh Z, Lam DY, Theodoro MF, Bittel DC, et al. (2006). Novel splice isoforms for NLGN3 and NLGN4 with possible implications in autism. J. Med. Genet. 43: e21.

Tao R, Yu Y, Zhang X, Guo Y, et al. (2005). Cytosolic PLA2 genes possibly contribute to the etiology of schizophrenia. Am. J. Med. Genet. B Neuropsychiatr. Genet. 137: 56-58.

Twiss JL and Fainzilber M (2009). Ribosomes in axons - scrounging from the neighbors? Trends Cell Biol. 19: 236-243.

Voineagu I, Wang X, Johnston P, Lowe JK, et al. (2011). Transcriptomic analysis of autistic brain reveals convergent molecular pathology. Nature 474: 380-384.

Wassink TH, Piven J, Vieland VJ, Huang J, et al. (2001). Evidence supporting WNT2 as an autism susceptibility gene. Am. J. Med. Genet. 105: 406-413.

Wei J, Lee KH and Hemmings GP (1998). Is the cPLA2 gene associated with schizophrenia? Mol. Psychiatry 3: 480-481. 